\title{
Effect of titrated parenteral nutrition on body composition after allogeneic hematopoietic stem cell transplantation in children: a double-blind, randomized, multicenter trial ${ }^{1-3}$
}

\author{
Tanvi S Sharma, Lori J Bechard, Henry A Feldman, Robert Venick, Kathleen Gura, Catherine M Gordon, Andrew Sonis, \\ Eva C Guinan, and Christopher Duggan
}

\begin{abstract}
Background: Children undergoing hematopoietic stem cell transplantation (HSCT) often require parenteral nutrition (PN) to optimize caloric intake. Standard approaches to nutritional supplementation provide $130-150 \%$ of estimated energy expenditure, but resting energy expenditure (REE) may be lower than expected after HSCT. Provision of PN exceeding energy needs may lead to overfeeding and associated complications.
\end{abstract}

Objective: We conducted a blinded, randomized, controlled, multicenter trial in children undergoing HSCT to determine the effect on body composition of 2 different approaches of nutrition support: standard amounts of energy from PN (130-150\% of REE) compared with PN titrated to match measured REE.

Design: Twenty-six children undergoing HSCT were randomly assigned to standard or titrated PN. Energy intake was monitored until day 30 after HSCT. Body-composition and anthropometric measures were obtained through day 100 . The primary outcome variable was percentage body fat $(\% \mathrm{BF})$ measured by dual energy X-ray absorptiometry.

Results: The estimated change in $\% \mathrm{BF}$ from baseline to day 30 was $1.2 \pm 0.5 \%$ in the standard group and $0.1 \pm 0.5 \%$ in the experimental group, but the overall time course of $\% \mathrm{BF}$ did not differ significantly by treatment $(P=0.39$ for time $\times$ treatment interaction). A profound loss of lean body mass (LBM) occurred in both groups during the intervention period and persisted through day 100.

Conclusions: Parenteral energy intake titrated to energy expenditure does not result in a lower accumulation of BF than does standard energy intake. Neither titrated nor standard PN regimens during HSCT preserve LBM. Alternative approaches to preserve LBM are needed. This trial is registered at clinicaltrials.gov as 00115258. Am J Clin Nutr 2012;95:342-51.

\section{INTRODUCTION}

\section{Scientific background and rationale}

Children undergoing $\mathrm{HSCT}^{4}$ are assumed to be in a catabolic state, and nutritional management of these children is primarily directed at optimizing caloric intake to keep up with ongoing energy expenditure. For children who have received intensive chemotherapy and total body irradiation in preparation for HSCT, painful mucositis and gastrointestinal sequelae may limit oral intake for days to weeks before and after transplantation. In this setting, $\mathrm{PN}$ is often required to ensure sufficient nutrient intake $(1,2)$. PN use has been associated with faster engraftment and improved survival in HSCT recipients $(3,4)$. Despite these advantages, PN use can also be associated with complications, including infections, hepatotoxicity, suppression of oral intake, and multiple metabolic abnormalities (5). Furthermore, there are potential risks with both overfeeding (6) and underfeeding (7) when supplementing or replacing oral intake with PN; however these risks have not been evaluated specifically in HSCT recipients.

Optimal PN support requires knowledge of the specific energy and nutritional needs of children undergoing HSCT. Studies of adults and children during HSCT have reported wide variability in REE, a clinically measurable approximation of BEE. These have included measurements both higher and lower than those estimated by standard equations (8-11). We previously showed a significant reduction of REE in children undergoing HSCT (12). In a cohort of 25 children undergoing allogeneic HSCT, we found that by week 3 after transplantation REE had declined to $80 \%$ predicted $(P=0.002)$. Furthermore, there was a significant decrease in midarm muscle area with no change in triceps skinfold thickness, which indicates preservation of fat stores but depletion of muscle mass.

As standard practice, children in the post-HSCT period are generally prescribed a PN regimen that provides $130-150 \%$ of

\footnotetext{
${ }^{1}$ From the Children's Hospital, Boston, Boston, MA (TSS, LJB, HAF, KG, CMG, AS, ECG, and CD); the Dana-Farber Cancer Institute, Boston, MA (LJB and ECG); and Mattel Children's Hospital at UCLA, Los Angeles, CA (RV).

${ }^{2}$ Supported by the Massachusetts Vitamin Litigation Grant; grant M01RR02172 from the NCRR, NIH to Children's Hospital, Boston GCRC; grant UL1 RR025758-01 to the Harvard Catalyst CTSA 1; grant M01-RR00865 to the General Clinical Research Centers Program at UCLA; and NIH K24 HD 058795 (to CD).

${ }^{3}$ Address correspondence and reprint requests to C Duggan, Division of GI/Nutrition, Children's Hospital, Boston, 300 Longwood Avenue, Boston, MA 02115. E-mail: christopher.duggan@ childrens.harvard.edu.

${ }^{4}$ Abbreviations used: ALT, alanine aminotransferase; BEE, basal energy expenditure; BF, body fat; DXA, dual-energy X-ray absorptiometry; GVHD, graft-versus-host disease; HSCT, hematopoietic stem cell transplantation; IV, intravenous; LBM, lean body mass; PN, parenteral nutrition; REE, resting energy expenditure; $\% \mathrm{BF}$, percentage body fat.

Received August 26, 2011. Accepted for publication November 18, 2011.

First published online December 28, 2011; doi: 10.3945/ajcn.111.026005.
} 
their estimated BEE (1), but our finding of a reduction in REE in HSCT recipients suggests that this amount of energy may be excessive. Limited data have also suggested that avoiding parenteral overfeeding may be beneficial during $\operatorname{HSCT}(13,14)$. These studies were nonrandomized comparisons of different compositions of PN among adults. Taveroff et al (13) studied 10 consecutive HSCT recipients who received $150 \%$ of estimated BEE and 5 subsequent patients who received only $100 \%$ of estimated BEE. The group that received a lower amount of parenteral energy had significantly higher serum albumin concentrations and a lower incidence of hyponatremia and hyperkalemia than did the group that received standard amounts of nutrition. Both groups had negative nitrogen balance, but this was not significantly different between groups. In 61 patients, Cetin et al (14) found that total $\left(30 \mathrm{kcal} \cdot \mathrm{kg}^{-1} \cdot \mathrm{d}^{-1}\right)$ PN led to higher blood urea and glucose concentrations, more frequent infections and delayed platelet engraftment compared with partial PN (5 $\mathrm{kcal} \cdot \mathrm{kg}^{-1} \cdot \mathrm{d}^{-1}$ ). Neither study provided detailed measures of energy expenditure, body composition, or longer follow-up in response to these PN regimens; however, these studies suggest that there is a need to study the potential effect of overfeeding in pediatric HSCT recipients.

\section{Objectives and hypothesis}

To evaluate whether the standard approach of providing nutrition support at $130-150 \%$ of predicted REE in children undergoing HSCT is associated with overfeeding and its associated complications, we performed a multicenter randomized controlled trial to compare the effects of standard nutritional support compared with nutritional support titrated to REE. The primary objective of this study was to determine the effect of the intervention on body composition, as measured by DXA. Secondary outcomes included evaluation of anthropometric and biochemical measures. We hypothesized that children receiving PN titrated to measured REE would have a smaller change in \% BF than would those who received standard parenteral energy intake.

\section{SUBJECTS AND METHODS}

\section{Trial design}

The study procedures were described in detail previously (15) and are summarized here. The study was a randomized, doubleblind, controlled clinical trial in which 2 approaches to providing PN to pediatric HSCT recipients were compared: 1) the standard approach in which energy intake is given at $130-150 \%$ of estimated BEE as calculated by standard reference equations (16), and 2) an experimental protocol in which energy intake was titrated to match REE as measured by indirect calorimetry. On the basis of the likelihood of corticosteroid exposure in children undergoing unrelated donor HSCT and the known influences of steroid use on body composition $(17,18)$, patients were randomly assigned within strata with respect to the type of donor (unrelated compared with related). All participants received nutritional support as clinically indicated and as described in detail below, but the energy goal of each group differed. The goal for participants in the standard arm was 140\% of estimated BEE, calculated by using the Schofield method (16). For par- ticipants in the experimental arm, the parenteral energy prescription was adjusted so that the energy goal was equal to $100 \%$ of the most recent measured REE. This was accomplished by alterations in dextrose and lipid; total protein goals were kept constant and did not differ between the 2 groups. Oral intake was subtracted from the total energy needs calculation in both groups; therefore, the target energy intake was determined by the sum of oral intake plus PN.

The study period was defined as the entire period of enrollment, from the initial screening period 7-14 d before HSCT through day 100 after HSCT. The intervention period was defined as the period from day 0 (the day of HSCT) to $30 \mathrm{~d}$. During the intervention period, participants whose oral intake fell below baseline REE for more than 3-5 d received PN with an energy goal in accordance with the arm to which the patient was randomly assigned (standard compared with experimental). Energy goals were managed by the study team only within the part of the intervention period during which PN was required. The study team did not manage energy goals after the participants resumed full oral intake or for patients who did not require $\mathrm{PN}$ at all. The follow-up period was defined as the period from 30 to $100 \mathrm{~d}$ after HSCT during which participants continued to be followed clinically and for study measures, but received no ongoing nutritional intervention.

\section{Study subjects and eligibility}

Children who were admitted for allogeneic HSCT at Children's Hospital Boston or UCLA Mattel Children's Hospital were eligible for this study. Children were considered for enrollment if they met the following inclusion criteria: 1) first myeloablative allogeneic HSCT using either total body irradiation (1400 cGy) or busulfan in addition to other chemotherapeutic agents, 2) GVHD prophylaxis with a calcineurin inhibitor and methotrexate with or without corticosteroids, 3) 6/6 human leukocyte antigen-matched and related or unrelated HSCT donor, and 4) age $\geq 6 \mathrm{y}$.

Children were excluded from enrollment for the following reasons: 1 ) age $<6$ y or otherwise unable to comply with study procedures, 2) underweight (BMI $z$ score $<-2$ for age and sex) at baseline, 3) overweight (BMI $z$ score $>2$ for age and sex) at baseline, 4) unable or unwilling to return for day 100 studies, 5) previous stem cell transplantation, 6) unmatched HSCT donor, 7) current treatment of hypo- or hyperthyroidism, 8) current insulin-dependent diabetes, 9) current use of PN, 10) allergy to egg or soy products, and 11) other contraindication to PN at baseline.

\section{Study interventions and protocol}

All enrolled participants received oncologic and medical care per institutional HSCT standard guidelines and protocols. Central venous catheters were used for administration of IV fluid and PN. In accordance with standard practice, PN was initiated if oral energy intake declined to less than baseline REE measurement for $>3-5 \mathrm{~d}$, but no earlier than the day of transplantation. Intravenous fluid from PN provided $\sim 50 \%$ of calculated maintenance fluid requirements, with the remainder of fluid intake provided from medications, blood products, oral intake, and other IV fluids as needed. Parenteral amino acids (20\% ProSol; 
Baxter International) were prescribed at $1.5-2.0 \mathrm{~g} \cdot \mathrm{kg}^{-1} \cdot \mathrm{d}^{-1}$ for 6-13-y-old children and $1.0-1.5 \mathrm{~g} \cdot \mathrm{kg}^{-1} \cdot \mathrm{d}^{-1}$ for children older than 13 y $(19,20)$. Intravenous lipids (Intralipid 20\%; Baxter International) provided $\sim 30-40 \%$ of total parenteral energy intake. Standard doses of pediatric (age 6-11 y) and adult (age $\geq 11$ y) intravenous multivitamins were supplied. A combination pediatric trace mineral solution, including zinc, copper, manganese, and chromium, was provided daily at standard doses. Electrolytes and minerals were monitored and adjusted daily by HSCT physicians according to laboratory results. The macronutrient composition of $\mathrm{PN}$ was titrated to match the total protein and energy goals of the participant's group assignment based on the previous day's (calculated twice weekly) oral protein and energy intakes. After engraftment and at the time of preparation for hospital discharge, PN was discontinued when oral energy intake reached $\geq 50 \%$ of the measured REE.

\section{Data collection and assessment of study outcomes}

Before conditioning, baseline study measures were obtained, including anthropometric measures, REE measurement, DXA, dietary intake assessment, and laboratory studies. In addition to the study measures, standardized demographic and clinical assessments of all study participants were performed by medical record review during the hospitalization and at clinical visits during the study and follow-up periods. Clinical assessments that were documented included medications, particularly corticosteroids, which are known to have an influence on body composition and glycemic control, presumed and confirmed infections, mucositis, and other factors associated with alterations in food intake, and GVHD.

Study measures included anthropometric assessments: body weight measured to the nearest $0.1 \mathrm{~kg}$; standing height measured to the nearest $0.1 \mathrm{~cm}$; triceps, biceps, iliac, and subscapular skinfold thicknesses measured to the nearest $0.5 \mathrm{~mm}$ with Lange skin calipers; and midarm muscle circumference measured to the nearest $0.1 \mathrm{~cm}$. Body density and \%BF were calculated from published equations by using skinfold-thickness measures (21). Skinfold-thickness measurements were standardized by using test subjects and comparing the results obtained by 2 study personnel. The technique was reviewed, and measurements were repeated until test measurements were within $10 \%$ of each other. All anthropometric measurements were obtained at baseline, day 30, and day 100 following HSCT. In addition, weight was measured daily to day 30 and also at day 65 . While in the hospital, the participants' oral intake was recorded through bedside calorie counts. Total intakes of calories, protein, fat, carbohydrates, and selected micronutrients were determined by using nutritional analysis software (Nutritionist Pro, ESHA Food Processor, and Nutrient Data Systems) and known specifications for PN solutions. Dietary intake was determined at baseline and daily through day 30. REE was measured by using the Vmax Encore indirect calorimeter (Viasys Health Care), and participants were studied in a modified fasted state that entailed $\geq 6 \mathrm{~h}$ without oral or enteral intake but receiving fluids and PN as needed. REE was measured at baseline, twice weekly, to day 30, at day 30, and at day 100. DXA was performed in all participants at baseline, day 30 , and day 100. This radiographic technique measures the composition, distribution, and density of different body com- partments, including fat mass, fat-free mass, and bone mineral content. The Hologic Discovery A (Hologic Inc) scanner was used at both study sites, and a certified densitometry technologist performed all DXA measurements. Body-composition data from DXA were sent quarterly to Children's Hospital Boston for centralized analysis and interpretation by using the Hologic Pediatric Upgrade for children and adolescents up to age $20 \mathrm{y}$. The DXA scanners were cross-calibrated with a single wholebody phantom shipped to UCLA. Weekly whole-body phantom scans were performed for quality control. Finally, biochemical assessments including measures of visceral protein status (albumin and prealbumin), electrolytes, and ALT were performed at baseline, day 30, and day 100.

\section{Power and sample size}

Power for this study was estimated on the basis of published data for variability of $\% \mathrm{BF}$ in children. Published norms put $\% \mathrm{BF}$ at roughly $28 \pm 8 \%$ (mean $\pm \mathrm{SD}$ ) in white and African American girls aged 6-17 and $18 \pm 9 \%$ in boys of the same age $(22,23)$. To determine the variance of change in $\% \mathrm{BF}$ from the variance of $\% \mathrm{BF}$ itself, we assumed the within-subject correlation to be 0.7 . A sample size of $n=20$ per arm provided $80 \%$ power to detect an absolute difference of $6 \%$ BF between arms with $P<0.05$ as the critical value in a 2 -sided test. On completion of the study, we used the realized sample size and SE to recalculate the difference detectable with $80 \%$ power as well as to calculate post hoc power for the hypothesized difference $(6 \%)$ and the observed estimate.

\section{Randomization and blinding}

A computer-generated sequence of treatment assignments was developed for each site in randomly permuted blocks of 2,4 , and 6. The specific sequence information was stored in locked cabinets by the Clinical Research Program at Children's Hospital Boston, which served as the data coordination site. Each list was divided into 2 balanced strata: 1 for related donors and 1 for unrelated donors. A separate enrollment log for each site, similarly numbered but without treatment assignments, was maintained by the study coordinator at each site.

The patients, parents, physicians, and nurses were blinded to treatment allocation and were therefore not informed of the energy composition of the PN. The pharmacist preparing the PN solution was unblinded but not directly involved in the conduct of the study. In addition, the registered dietitian of the clinical team was unblinded and was responsible for calculating each participant's regimen and writing fluid and macronutrient orders using the standard or experimental method depending on the participant's group designation. On enrollment, this unblinded dietitian entered the patient into the randomization log, thus committing the patient to that position in the randomization sequence. This ordering dietitian was responsible for clinical care but not for study measurements.

\section{Data and safety monitoring}

Adverse events were reported as disease entities or syndromes and classified according to the National Cancer Institute Common Toxicity Criteria, v3.0 (24). The occurrence of significant weight loss ( $>15 \%$ of admission weight) or low visceral protein status 
(albumin $<1.5 \mathrm{~g} / \mathrm{dL}$ or prealbumin $<10 \mathrm{mg} / \mathrm{dL}$ ) after $7 \mathrm{~d}$ of $\mathrm{PN}$ were considered serious adverse events and were regarded as possible evidence of inadequate PN. The Institutional Review Boards at both institutions approved the protocol and procedures for informed consent and assent were followed.

\section{Statistical methods}

Baseline characteristics and clinical outcomes were summarized by standard descriptive statistics and compared between treatment arms by Fisher's exact test for categorical variables and Student's independent $t$ test for continuous measures, corroborated by the Wilcoxon's signed-rank sum test in the case of skewed distributions. The time course of caloric intake in each treatment arm was summarized by means and SEs on each study day and also by the mean of subjects' average levels over days 0-30. The time course was compared longitudinally between arms by smoothing spline analysis by using $12 \mathrm{kn}$ spaced at $3-\mathrm{d}$ intervals between day -6 and day 27 and a truncated power basis of degree 1. The spline-fitting procedure was implemented in SAS by use of PROC MIXED with the use of the equivalent random-effects formulation of the linear penalized spline model (25).

The primary endpoint of the trial, \%BF from DXA, was analyzed by repeated-measures ANOVA of the baseline, 30-d, and 100-d measurements by using an unconstrained covariance structure. The independent variables were time, treatment arm, and time $\times$ treatment interaction, with covariate adjustments for age, sex, and donor type (related or unrelated). An alternative model with adjustment for steroid dosage produced similar results. From parameters of the fitted ANOVA model, we constructed the following quantities of interest: adjusted mean at baseline, $30 \mathrm{~d}$, and $100 \mathrm{~d}$; 30-d change in each treatment arm; difference between arms in the magnitude of 30-d change; 100-d change in each treatment arm; and difference between arms in the magnitude of 100-d change. Other anthropometric, bodycomposition, and laboratory measures were analyzed similarly. SAS software (version 9.2; SAS Institute) was used for all computations.

For the primary analysis, we used the intention-to-treat principle, classifying each subject in the randomly assigned treatment group regardless of compliance. In an alternative secondary analysis, we omitted data from the 3 subjects ( 2 experimental, 1 standard) who received no $\mathrm{PN}$ due to adequate oral intake throughout the course of HSCT.

To avoid spurious inference from multiple comparisons, we restricted hypothesis testing to the time $\times$ treatment interaction, addressing in a single test the question of differential effect of the standard and experimental PN regimes on the time course of each measure. Statistical significance was taken as $P<0.05$ for each measure, making the Type I error rate $5 \%$ for the primary endpoint $(\% \mathrm{BF})$ and the expected number of Type I errors $<1$ for the set of 16 anthropometric and body-composition measures reported here.

\section{RESULTS}

The participants were enrolled for this study from February 2004 to February 2009. Participant enrollment, allocation, and follow-up during the study period (Consolidated Standards of Reporting Trials; 26) are shown in Figure 1. Of 90 eligible patients scheduled for HSCT, 26 agreed to enrollment in the study and were randomly assigned to the standard or experimental nutritional care arms. The most common reason provided for refusal to participate was the desire of parents to limit additional interventions and measurements during HSCT. Enrollment ended before our planned sample size was attained because of difficulty with participant recruitment and budgetary limitations.

Participant demographic characteristics and clinical diagnoses are described in Table 1. Age at enrollment, sex, underlying

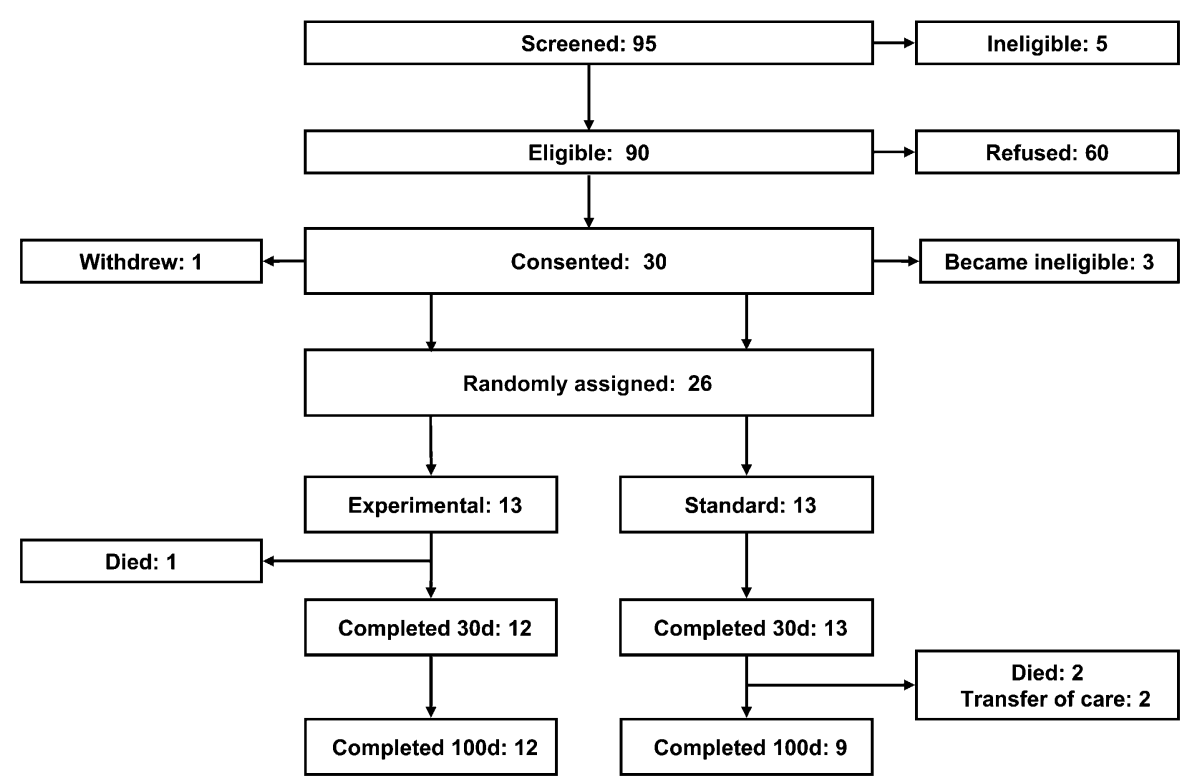

FIGURE 1. Flow diagram depicting enrollment, allocation, and follow-up of study participants. Two patients in the experimental group and 1 patient in the standard group did not ultimately require parenteral nutrition, but their data were nonetheless collected and included in an intention-to-treat analysis as if they had received the assigned treatment. 
diagnoses, donor type, anthropometric and body-composition measures (including mean height, weight, BMI, and \%BF by DXA), and dietary and energy measures, including oral intake, protein intake, and measured REE, were similar in both the standard and experimental arms. Pretransplantation corticosteroid use was noted in 7 subjects in the standard group and 3 in the experimental group.

The difference in total intake between the standard and experimental arms of the study are shown in Figure 2, which indicates that the nutrition support provided to each group of patients was consistent with their group assignment. Spline analysis confirmed this difference between treatment arms $(P=$ 0.006 for total intake, $P<0.0001$ for excess over REE). We further analyzed data on oral energy intake, total protein and amino acid intake, and PN/IV energy intake in the standard compared with the experimental groups until day 30. No significant difference in mean $( \pm \mathrm{SE})$ daily oral intake was observed over the first $30 \mathrm{~d}$ in the standard group compared with the experimental group (standard: $477.7 \pm 167.0 \mathrm{kcal}$; experimental: $444.8 \pm 99.9 \mathrm{kcal} ; P=0.93$ from spline analysis). Similarly, over the same time frame, no difference was found in total protein intake (standard: $55.4 \pm 5.3 \mathrm{~g}$; experimental: $53.2 \pm$ $4.6 \mathrm{~g} ; P=0.85$ ), oral protein intake (standard: $12.9 \pm 4.3 \mathrm{~g}$; experimental: $11.7 \pm 3.6 \mathrm{~g} ; P=0.91$ ), and $\mathrm{PN}$ amino acid intake (standard: $42.5 \pm 6.2 \mathrm{~g}$; experimental: $41.6 \pm 6.8 \mathrm{~g} ; P=0.94$ ) between the 2 study arms. The only significant difference that was noted was in the total PN/IV energy intake between the groups (standard: $1304.4 \pm 174.7 \mathrm{kcal}$; experimental: $820.8 \pm$
$125.4 \mathrm{kcal} ; P<0.0001$ ), which was consistent with our study design.

The changes in anthropometric and body-composition measures in both the standard and experimental groups, from baseline to $30 \mathrm{~d}$ and $100 \mathrm{~d}$ after HSCT, adjusted for age, sex, and donor type are shown in Table 2. Anthropometric measures declined over the $30 \mathrm{~d}$ after transplantation, including weight, weight-forage $z$ score, and, in the experimental group, BMI and BMI $z$ score. None of these changes differed significantly between the treatment groups. Weight and BMI declined further in both groups at $100 \mathrm{~d}$.

Changes in \%BF over the course of the study are shown in Figure 3. The estimated change in $\% \mathrm{BF}$ in the standard group was $1.2 \pm 0.5 \%$ (mean $\pm \mathrm{SE}$ ) from baseline to day 30 , whereas in the experimental group the estimated change was $0.1 \pm 0.5 \%$. A similar pattern was observed at day 100. The time course did not differ significantly between groups $(P=0.39$ for time $\times$ treatment interaction).

As also noted in Table 2, LBM decreased substantially from baseline in both groups, and this decrease persisted beyond the intervention period through day 100 (standard: $-3.84 \pm 0.97 \mathrm{~kg}$; experimental: $-3.29 \pm 0.92 \mathrm{~kg}$ ). No significant difference was found between the standard and experimental groups in degree of LBM loss over the course of the study $(P=0.18$ for time $\times$ treatment interaction). Other body-composition measures, including muscle mass as estimated by midarm muscle area and skinfold thicknesses, decreased in all patients in the post-HSCT period, but the changes did not differ significantly between treatment groups.

TABLE 1

Baseline characteristics of the study participants ${ }^{1}$

\begin{tabular}{lcc}
\hline \hline & Standard $(n=13)$ & Experimental $(n=13)$ \\
\hline Age $(\mathrm{y})$ & $15.1 \pm 4.1^{2}$ & $14.7 \pm 4.5$ \\
Female sex $[n(\%)]$ & $8(62)$ & $6(46)$ \\
Nonwhite race $[n(\%)]$ & $6(46)$ & $5(38)$ \\
Diagnosis $[n(\%)]$ & & \\
$\quad$ Acute lymphoblastic leukemia & $3(23)$ & $4(31)$ \\
Acute myelogenous leukemia & $4(31)$ & $3(23)$ \\
Myelodysplastic syndrome & $2(15)$ & $1(8)$ \\
Aplastic anemia & $0(0)$ & $1(8)$ \\
Chronic myelogenous leukemia & $2(15)$ & $1(8)$ \\
Lymphoma & $1(8)$ & $1(8)$ \\
Other & $1(8)$ & $2(15)$ \\
Donor type $[n(\%)]$ & & $6(46)$ \\
Sibling/related & $6(46)$ & $7(54)$ \\
Unrelated & $7(54)$ & $153 \pm 22$ \\
Anthropometric/body-composition measures & & $51.0 \pm 19.0$ \\
Height $(\mathrm{cm})$ & $156 \pm 17$ & $20.8 \pm 3.6$ \\
Weight $(\mathrm{kg})$ & $54.2 \pm 17.2$ & $0.31 \pm 0.80$ \\
BMI $\left(\mathrm{kg} / \mathrm{m}^{2}\right)$ & $21.4 \pm 3.8$ & $28.4 \pm 9.0$ \\
BMI $(z$ score) & $0.31 \pm 0.89$ & $1918 \pm 384$ \\
Body fat by DXA $(\%)$ & $29.2 \pm 8.2$ & $80 \pm 37$ \\
Diet and energy measures & & $1259 \pm 326$ \\
Oral intake at baseline $(\mathrm{kcal} / \mathrm{d})$ & $2127 \pm 1084$ & $72 \pm 33$ \\
Protein intake $(\mathrm{g} / \mathrm{d})$ & $1366 \pm 317$ & \\
REE at baseline $(\mathrm{kcal} / \mathrm{d})$ & &
\end{tabular}

${ }^{1}$ The mean value of each continuous variable was compared between trial arms by Student's $t$ test, corroborated by Wilcoxon's 2-sample test in cases of skewed distribution. The distribution of each discrete variable was compared by Fisher's exact test. All differences were nonsignificant, $P>0.40$. DXA, dual-energy X-ray absorptiometry; REE, resting energy expenditure.

${ }^{2}$ Mean $\pm \mathrm{SD}$ (all such values). 

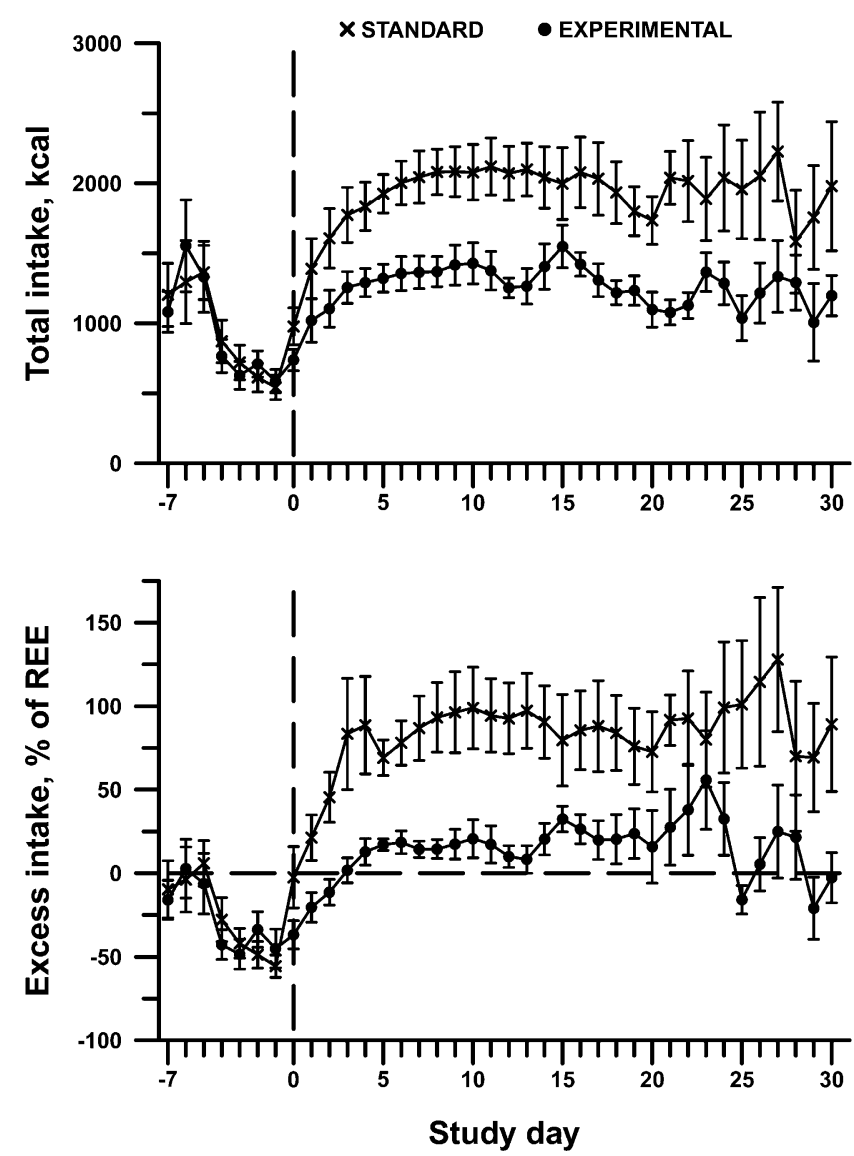

FIGURE 2. Dietary intake immediately preceding transplant (study day 0 ) declined by $\sim 50 \%$. Over $30 \mathrm{~d}$ of hospitalization after transplant, subjects randomly assigned to the standard nutrition protocol received nearly twice their REE, whereas those randomly assigned to the experimental protocol were maintained closely to caloric requirements. Data are represented as unadjusted means \pm SEs; $n=12-13$ subjects per treatment group on each day through day 20 , then 5-12 per group through day 30 . Linear spline analysis indicated that the time course for the 2 treatment groups diverged significantly for both total caloric intake (upper panel; $P=0.007$ ) and excess over REE (lower panel; $P<0.0001$ ). REE, resting energy expenditure.

We further analyzed DXA measurements to compare regional changes in body composition in the 2 treatment groups, finding some changes but no significant treatment differences (Table 2). Peripheral (extremity) LBM decreased in both groups from baseline (standard: $-1.31 \pm 0.43 \mathrm{~kg}$ at day $30,-2.39 \pm 0.58 \mathrm{~kg}$ at day 100; experimental: $-1.85 \pm 0.43$ at day $30,-2.05 \pm 0.56$ $\mathrm{kg}$ at day 100). Peripheral fat mass also decreased in both groups, to a lesser extent. Central (trunk) fat and central LBM changed little, whereas peripheral fat increased by $1-2 \%$ in both groups.

Three study participants ( 2 in the experimental group and 1 in the standard group) did not require PN during the intervention period. In a secondary "as-treated" analysis among the remaining 23 subjects, the results for $\% \mathrm{BF}$ and fat mass were similar to those obtained from analysis of the full sample.

Clinical outcomes, including mortality, infections, length of hospitalization, and development of GVHD, were similar in both groups of patients during the study period and are shown in Table 3. All children in both arms achieved sufficient oral intake to be able to discontinue PN by $\sim 30 \mathrm{~d}$ after HSCT.
The time course of laboratory assessments, including glucose, insulin, C-peptide, triglycerides, sodium, potassium, bicarbonate, ALT, blood urea nitrogen, and creatinine, did not differ significantly between the groups over the 30 -d period of intervention (data not shown).

Serum triglycerides increased in both groups over the course of the study, in the standard group from $109 \pm 17 \mathrm{mg} / \mathrm{dL}$ at baseline (mean $\pm \mathrm{SE}$ ) to $267 \pm 35 \mathrm{mg} / \mathrm{dL}$ at day 30 and to $227 \pm 37 \mathrm{mg} /$ $\mathrm{dL}$ at day 100; in the experimental group, from $102 \pm 16 \mathrm{mg} / \mathrm{dL}$ at baseline to $204 \pm 38 \mathrm{mg} / \mathrm{dL}$ at day 30 and to $176 \pm 21 \mathrm{mg} / \mathrm{dL}$ at day 100. The pattern of increase did not differ significantly between the groups ( $P=0.46$ for time $\times$ treatment interaction). No adverse events associated with treatment (eg, $>15 \%$ weight loss, hypoalbuminemia) were noted in either group during the intervention period.

Post hoc power analysis was based on our observed primary endpoint - the difference between groups in the mean 30-d change in $\% \mathrm{BF}$, estimated at $1.028 \pm 0.735 \%$ (estimate $\pm \mathrm{SE}$ adjusted for age and donor type). This realized SE reflected the attained sample size (13 per group rather than the planned 20 per group) as well as the actual endpoint variability and intrasubject correlation, which were a matter of informed conjecture in the design of the trial. Using these parameters we calculated that the study carried $>99 \%$ power to detect an effect of the hypothesized magnitude $(6 \% \mathrm{BF}) ; 80 \%$ power to detect an effect of $2.2 \%$ (thus nearly 3 -fold more sensitive than originally estimated); and $25 \%$ power to detect an underlying effect of the magnitude observed $(1.0 \%)$.

\section{DISCUSSION}

In this randomized, blinded trial of 2 levels of energy intake in children undergoing HSCT, we determined that titrating energy intake to REE was not associated with significant improvement in body composition compared with standard energy prescription. Regardless of energy intake during the first $30 \mathrm{~d}$ after transplantation, patients exhibited no significant differences in either $\%$ BF or LBM. We did, however, note substantial declines in LBM, ranging from 1.6 to $2.5 \mathrm{~kg}$ by day 30 and $3.3-3.8 \mathrm{~kg}$ by day 100.

We found that regional distribution of BF was not different between the standard and experimental groups, but there was a reduction in peripheral lean mass in both groups and relative preservation of central fat. Central adiposity is known to be associated with greater cardiometabolic complications in the long term, such as insulin resistance, and may be a more important factor than total BF in evaluating nutritional needs and the potential risk of overfeeding in HSCT recipients $(27,28)$. Therefore regional differences in $\mathrm{BF}$ distribution in this population merit ongoing follow-up.

A profound loss of LBM was seen in patients in this study, which was consistent with the findings in our previous study, in which LBM was estimated by measure of arm anthropometric measures (12). In a different patient population, Streat et al (29) evaluated nutritional intake and body composition in adult patients admitted to an intensive care unit with septic shock. They found that patients lost $12.5 \%$ of their total body protein within days of their acute illness, and that this protein loss occurred despite aggressive nutritional support that included nearly 3000 
TABLE 2

Change in anthropometric and body-composition measures from transplant (baseline) to day 30 and day $100^{l}$

\begin{tabular}{|c|c|c|c|c|}
\hline Treatment & Baseline $^{2}$ & $\begin{array}{c}\text { Change, day } \\
30^{3,4}\end{array}$ & $\begin{array}{c}\text { Change, day } \\
100^{4,5}\end{array}$ & $P^{6}$ \\
\hline \multicolumn{5}{|c|}{ Anthropometric measures } \\
\hline \multicolumn{5}{|c|}{ Weight $(\mathrm{kg})$} \\
\hline Standard & $54.2 \pm 4.8$ & $-2.1 \pm 0.9$ & $-4.5 \pm 1.7$ & \multirow[t]{2}{*}{0.57} \\
\hline Experimental & $51.0 \pm 5.3$ & $-3.3 \pm 0.9$ & $-4.6 \pm 1.6$ & \\
\hline \multicolumn{5}{|l|}{ Weight $z$ score } \\
\hline Standard & $0.19 \pm 0.27$ & $-0.19 \pm 0.08$ & $-0.61 \pm 0.23$ & \multirow[t]{2}{*}{0.39} \\
\hline Experimental & $-0.07 \pm 0.30$ & $-0.35 \pm 0.08$ & $-0.49 \pm 0.20$ & \\
\hline \multicolumn{5}{|l|}{ BMI $\left(\mathrm{kg} / \mathrm{m}^{2}\right)$} \\
\hline Standard & $21.4 \pm 1.1$ & $-0.6 \pm 0.4$ & $-1.3 \pm 0.7$ & \multirow[t]{2}{*}{0.39} \\
\hline Experimental & $20.8 \pm 1.0$ & $-1.3 \pm 0.4$ & $-1.5 \pm 0.6$ & \\
\hline \multicolumn{5}{|l|}{ BMI $z$ score } \\
\hline Standard & $0.31 \pm 0.25$ & $-0.14 \pm 0.12$ & $-0.59 \pm 0.27$ & \multirow[t]{2}{*}{0.29} \\
\hline Experimental & $0.31 \pm 0.22$ & $-0.42 \pm 0.13$ & $-0.52 \pm 0.23$ & \\
\hline \multicolumn{5}{|c|}{ Body-composition measures } \\
\hline Total body fat $(\%$ & & & & \\
\hline Standard & $29.2 \pm 2.3$ & $1.2 \pm 0.5$ & $1.3 \pm 1.6$ & \multirow[t]{2}{*}{0.39} \\
\hline Experimental & $28.4 \pm 2.5$ & $0.1 \pm 0.5$ & $0.4 \pm 1.5$ & \\
\hline \multicolumn{5}{|c|}{ Total body fat (\% calculated from skinfolds) } \\
\hline Standard & $28.6 \pm 2.0$ & $-0.8 \pm 0.7$ & $-1.6 \pm 1.6$ & \multirow[t]{2}{*}{0.57} \\
\hline Experimental & $26.0 \pm 1.9$ & $-1.1 \pm 0.7$ & $0.0 \pm 1.5$ & \\
\hline \multicolumn{5}{|c|}{ Total body fat $(\mathrm{kg})$} \\
\hline Standard & $15.94 \pm 1.99$ & $0.07 \pm 0.29$ & $-0.42 \pm 1.29$ & \multirow[t]{2}{*}{0.16} \\
\hline Experimental & $14.36 \pm 2.05$ & $-0.65 \pm 0.30$ & $-1.23 \pm 1.21$ & \\
\hline \multicolumn{5}{|l|}{ Total LBM (kg) } \\
\hline Standard & $36.36 \pm 3.56$ & $-1.61 \pm 0.70$ & $-3.84 \pm 0.97$ & \multirow[t]{2}{*}{0.18} \\
\hline Experimental & $34.48 \pm 3.94$ & $-2.48 \pm 0.71$ & $-3.29 \pm 0.92$ & \\
\hline \multicolumn{5}{|l|}{ Central fat $(\%)$} \\
\hline Standard & $24.9 \pm 2.6$ & $0.8 \pm 0.6$ & $1.7 \pm 1.9$ & \multirow[t]{2}{*}{0.22} \\
\hline Experimental & $24.9 \pm 2.7$ & $-0.8 \pm 0.6$ & $0.5 \pm 1.7$ & \\
\hline \multicolumn{5}{|l|}{ Central fat (kg) } \\
\hline Standard & $6.57 \pm 1.08$ & $0.07 \pm 0.17$ & $-0.15 \pm 0.74$ & \multirow[t]{2}{*}{0.18} \\
\hline Experimental & $5.87 \pm 1.01$ & $-0.34 \pm 0.18$ & $-0.37 \pm 0.69$ & \\
\hline \multicolumn{5}{|c|}{ Central LBM (kg) } \\
\hline Standard & $18.39 \pm 1.82$ & $-0.35 \pm 0.32$ & $-1.42 \pm 0.52$ & \multirow[t]{2}{*}{0.55} \\
\hline Experimental & $16.88 \pm 1.95$ & $-0.62 \pm 0.33$ & $-1.25 \pm 0.49$ & \\
\hline Peripheral fat $(\%$ & & & & \\
\hline Standard & $35.0 \pm 2.5$ & $2.0 \pm 0.7$ & $1.4 \pm 1.9$ & 0.76 \\
\hline Experimental & $33.6 \pm 2.8$ & $1.3 \pm 0.7$ & $0.6 \pm 1.7$ & \\
\hline Peripheral fat $(\mathrm{k}$ & & & & \\
\hline Standard & $8.53 \pm 0.99$ & $-0.03 \pm 0.15$ & $-0.34 \pm 0.57$ & 0.43 \\
\hline Experimental & $7.67 \pm 1.06$ & $-0.32 \pm 0.16$ & $-0.86 \pm 0.52$ & \\
\hline Peripheral LBM & & & & \\
\hline Standard & $15.07 \pm 1.69$ & $-1.31 \pm 0.43$ & $-2.39 \pm 0.58$ & 0.06 \\
\hline Experimental & $14.69 \pm 1.90$ & $-1.85 \pm 0.43$ & $-2.05 \pm 0.56$ & \\
\hline Midarm muscle & & & & \\
\hline Standard & $33.5 \pm 2.9$ & $-1.5 \pm 1.2$ & $-2.5 \pm 1.5$ & 0.53 \\
\hline Experimental & $34.2 \pm 3.8$ & $-2.3 \pm 1.2$ & $-4.9 \pm 1.4$ & \\
\hline
\end{tabular}

${ }^{1} n=13$ subjects in each treatment group at baseline. LBM, lean body mass.

${ }^{2}$ Unadjusted means \pm SEs.

${ }^{3} n=12-13$ in the standard group and $10-12$ in the experimental group.

${ }^{4}$ Mean change from baseline \pm SE from repeated-measures ANOVA adjusted for age, sex, and type of donor.

${ }^{5} n=9$ in the standard group and 12 in the experimental group.

${ }^{6}$ Represents the comparison of time courses between the standard and experimental treatment groups (time $\times$ treatment interaction).

$\mathrm{kcal} / \mathrm{d}$ and $127 \mathrm{~g}$ amino acids. However, even with the profound protein loss, patients were noted to gain fat during the study period (29). Our findings similarly suggest that greater energy intake with a standard PN regimen does not help to maintain LBM; loss of LBM occurred in both study arms to a similar degree and persisted beyond the intervention period. These findings are particularly important when we consider that the goal of standard PN regimens has been to help prevent wasting during times of stress and illness. Studies in which nutritional interventions were aimed at increasing muscle mass by increasing 


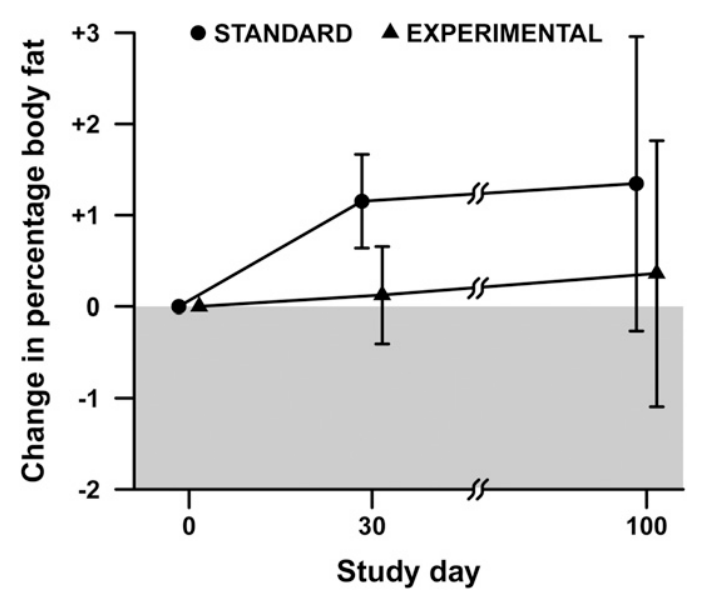

FIGURE 3. Change in percentage body fat from transplant (baseline) through hospitalization (day 30) and follow-up (day 100). Data are represented as means \pm SEs adjusted for age, sex, and type of donor; $n=$ 13 subjects in each treatment group at baseline, 12 standard and 11 experimental subjects at day 30 , and 9 standard and 12 experimental subjects at day 100. Repeated-measures ANOVA indicated no significant difference in time course between treatments $(P=0.39$ for time $\times$ treatment interaction).

protein intake have presented conflicting results, showing either modest benefits $(30,31)$ or no benefit at all (29). Furthermore, the potential effect of a higher intake of amino acids on tumor growth in cancer patients has been raised as a concern in animal models and small human studies (31) and may be an additional consideration in HSCT recipients.

Nonnutritional interventions to preserve LBM may have greater promise. For example, Jarden et al (32) evaluated a multimodal intervention of exercise, resistance training, and relaxation on psychological and physiologic outcomes, particularly muscle strength, and found that such interventions did improve muscle strength and reduce the need for PN in HSCT recipients. In a study of 7 children who had undergone HSCT, Chamorro-Viña et al (33) showed that a 3-wk in-hospital exercise intervention resulted in a modest increase in LBM when compared with children who did not receive the exercise intervention. Outpatient exercise interventions, weeks after HSCT, may also be beneficial; however, the direct effect on body composition of these latter interventions is unclear (34). Overall, these studies suggest that exercise may be an important adjunct for improving bodycomposition values in HSCT recipients and should be explored further in this population.

Our study did not indicate any adverse clinical outcomes in patients receiving titrated $\mathrm{PN}$ compared with the standard regimen. It is possible that the provision of higher amounts of parenteral energy might have increased BF more than we observed. The actual mean ( $\pm \mathrm{SD}$ ) energy intake in the standard group was $177 \pm 56 \%$ REE over the course of $30 \mathrm{~d}$. In our patients, REE dropped faster than anticipated, and the additional intake reflects the lag in modification of energy intake in between REE measurements. In standard practice, where REE is not measured routinely and PN intake is calculated according to standard formulas, it is possible that patients are receiving significantly higher energy intakes than the prescribed 130-150\% of the presumed REE, which may lead to additional concerns with overfeeding. Nonetheless, at the levels of energy intake we prescribed, no significant changes were noted.

Our data are consistent with those of recent studies of nutrition supplementation in critically ill patients where the concept of "permissive underfeeding" has emerged, in which energy intake is reduced to $60-70 \%$ of standard parenteral regimens. Reductions in mortality, length of hospitalization, septic complications, and improved glucose homeostasis have been reported, compared with standard feeding regimens (35-37). Our study did not observe, nor was powered, to detect these outcomes.

Our study was limited by the small number of patients who agreed to participate after initial eligibility screening. However, despite the small sample size, we had adequate power to detect a small $(2.2 \%)$ effect on BF. In addition, we did not systematically assess physical activity in our participants during the intervention period, particularly strength and resistance activities. Given the potential benefit on improving loss of LBM in HSCT recipients, studies directed at further evaluating the effect of physical activity in these children are needed.

Our findings require careful consideration in HSCT recipients. Whereas mortality and morbidity from HSCT have generally declined over time (38), the metabolic syndrome may be a longterm concern in survivors of childhood acute lymphoblastic leukemia treated with total body irradiation-based HSCT (39). Dyslipidemias, which are often related to chemotherapeutic medications, have been described in $59 \%$ of children treated with HSCT for leukemia at $<3$ y of age (40). Survivors of pediatric

TABLE 3

Clinical outcomes of the study participants ${ }^{1}$

\begin{tabular}{lcc}
\hline \hline & Standard & Experimental \\
\hline Mortality $[n(\%)]$ & $2(15)$ & $1(8)$ \\
Engraftment time (d) & $24 \pm 5^{2}$ & $24 \pm 6$ \\
Patients with confirmed infections during follow-up period $[n(\%)]$ & $6(46)$ & $4(31)$ \\
No. of infections & $0.6 \pm 0.9$ & $1.1 \pm 2.3$ \\
Patients with GVHD during follow-up period $[n(\%)]$ & $4(31)$ & $1(8)$ \\
Patients with veno-occlusive disease [n $(\%)]$ & $1(8)$ & $1(8)$ \\
Length of hospital stay (d) & $42 \pm 12$ & $39 \pm 10$ \\
\hline
\end{tabular}

${ }^{1}$ Mean values of each continuous variable were compared between trial arms by Student's $t$ test, corroborated by Wilcoxon's 2-sample test in cases of skewed distribution. The distribution of each binary variable was compared by Fisher's exact test. All differences were nonsignificant, $P>0.30$. GVHD, graft-versus-host disease.

${ }^{2}$ Mean \pm SD (all such values). 
cancer have significantly higher risks of cardiovascular disease (41) and obesity (42) than do their siblings. We observed reductions in LBM and elevations in triglyceride concentrations during the first $100 \mathrm{~d}$ after HSCT, which may be important contributors to these adverse metabolic outcomes. The results of our trial, however, do not implicate a role for parenteral energy intake in these changes.

In summary, important body-composition changes occur in HSCT recipients given nutritional support. Titrating energy intake to approximate REE does not result in significantly less BF accretion compared with the provision of standard amounts of energy. Other interventions aimed at preserving LBM should also be a component of the overall approach to improving nutrition for children undergoing HSCT.

We appreciate the valuable contributions of our subjects and families and the helpful assistance of Janet Mooney, Elena Khanukova, Nicolle Quinn, and Melissa O'Connor.

The authors' responsibilities were as follows-TSS: directly involved in the data analysis, data interpretation, and writing of the final manuscript; LJB: directly involved in the study design, study implementation and follow-up, data collection, data analysis, and data interpretation and assisted with the writing of the final manuscript; HAF: assisted with the study design, data interpretation, and writing of the final manuscript and conducted the data analysis; RV, KG, CMG, and AS: directly involved with the study implementation, data collection, and assistance with writing and reviewing the final manuscript; ECG: directly involved with the study design and implementation, data collection, and assistance with writing and reviewing the final manuscript; and CD (Principal Investigator): primarily responsible for obtaining funding, directly involved in study design, study implementation and followup, data collection, data analysis, data interpretation, assistance with writing of final manuscript, overall review and guidance in manuscript preparation. None of the authors had a conflict of interest.

\section{REFERENCES}

1. Muscaritoli M, Grieco G, Capria S, Iori AP, Rossi Fanelli F. Nutrition and metabolic support in patients undergoing bone marrow transplantation. Am J Clin Nutr 2002;75:183-90.

2. Bechard LJ, Guinan EC, Feldman HA, Tang V, Duggan C. Prognostic factors in the resumption of oral dietary intake after allogeneic hematopoietic stem cell transplantation (HSCT) in children. JPEN J Parenter Enteral Nutr 2007;31:295-301.

3. Weisdorf S, Hofland C, Sharp H, Teasley K, Schissel K, McGlave P, Ramsay N, Kersey J. Total parenteral nutrition in bone marrow transplantation: a clinical evaluation. J Pediatr Gastroenterol Nutr 1984;3: 95-100.

4. Weisdorf SA, Lysne J, Wind D, Haake R, Sharp H, Goldman A, Schissel K, McGlave PB, Ramsay NK, Kersey JH. Positive effect of prophylactic total parenteral nutrition on long-term outcome of bone marrow transplantation. Transplantation 1987;43:833-8.

5. Kerner JA Jr, Hurwitz M. Parenteral nutrition. In: Duggan C, Watkins J, Walker WA, eds. Nutrition in pediatrics: basic science and clinical applications. 4th ed. Hamilton, Ontario: BC Decker, 2008:777-93.

6. Chwals WJ. Overfeeding the critically ill child: fact or fantasy? New Horiz 1994;2:147-55.

7. Goulet O. Assessment of nutritional status in clinical practice. Baillieres Clin Gastroenterol 1998;12:647-69.

8. Hutchinson ML, Clemans GW, Springmeyer SC, Flournoy N. Energy expenditure estimation in recipients of marrow transplants. Cancer 1984;54:1734-8.

9. Chamouard Cogoluenhes V, Chambrier C, Michallet M, Gordiani B, Ranchere JY, Combret D, Lallemand Y, Roldan M, Accominoti M, Philip I, et al. Energy expenditure during allogeneic and autologous bone marrow transplantation. Clin Nutr 1998;17:253-7.

10. Yamanaka H, Takeda E, Takata K, Syutou E, Miyamoto K, Watanabe T, Kawano Y, Takaue Y, Kuroda Y. Total parenteral nutrition on energy metabolism in children undergoing autologous peripheral blood stem cell transplantation. J Med Invest 1998;44:199-203.
11. Ringwald-Smith KA, Heslop HE, Krance RA, Mackert PW, Hancock ML, Stricklin LM, Bowman LC, Hale GA. Energy expenditure in children undergoing hematopoietic stem cell transplantation. Bone Marrow Transplant 2002;30:125-30.

12. Duggan C, Bechard L, Donovan K, Vangel M, O’Leary A, Holmes C, Lehmann L, Guinan E. Changes in resting energy expenditure among children undergoing allogeneic stem cell transplantation. Am J Clin Nutr 2003;78:104-9.

13. Taveroff A, McArdle AH, Rybka WB. Reducing parenteral energy and protein intake improves metabolic homeostasis after bone marrow transplantation. Am J Clin Nutr 1991;54:1087-92.

14. Cetin T, Arpaci F, Dere Y, Turan M, Ozturk B, Komurcu S, Ozet A, Beyzadeoglu M, Kaptan K, Beyan C, et al. Total parenteral nutrition delays platelet engraftment in patients who undergo autologous hematopoietic stem cell transplantation. Nutrition 2002;18:599-603.

15. Bechard LJ, Feldman H, Gordon C, Gura K, Sonis A, Leung K, Venick R, Guinan EC, Duggan C. A multi-center, randomized, controlled trial of parenteral nutrition titrated to resting energy expenditure in children undergoing hematopoietic stem cell transplantation ("PNTREE"): rationale and design. Contemp Clin Trials 2010;31:157-64.

16. Schofield WN. Predicting basal metabolic rate, new standards and review of previous work. Hum Nutr Clin Nutr 1985;39(suppl 1):5-41.

17. Cowan FJ, Warner JT, Dunstan FD, Evans WD, Gregory JW, Jenkins HR. Inflammatory bowel disease and predisposition to osteopenia. Arch Dis Child 1997;76:325-9.

18. Mul D, van Suijlekom-Smit LW, ten Cate R, Bekkering WP, de Muinck Keizer-Schrama SM. Bone mineral density and body composition and influencing factors in children with rheumatic diseases treated with corticosteroids. J Pediatr Endocrinol Metab 2002;15:187-92.

19. Noel R, Udall J. Parenteral nutrition. In: Walker W, Watkins JB, eds. Nutrition in pediatrics: basic science and clinical applications. 2nd ed. Hamilton, Ontario: BC Decker, 1996:734-46.

20. Collier S, Gura K, Richardson D, Duggan C. Parenteral nutrition. In: Hendricks K, Duggan C, eds. Manual of pediatric nutrition. 4th ed. Hamilton, Ontario: BC Decker, 2005:317-75.

21. Zemel BS, Riley EM, Stallings VA. Evaluation of methodology for nutritional assessment in children: anthropometry, body composition, and energy expenditure. Annu Rev Nutr 1997;17:211-35.

22. Ellis KJ, Abrams SA, Wong WW. Body composition of a young, multiethnic female population. Am J Clin Nutr 1997;65:724-31.

23. Ellis KJ. Body composition of a young, multiethnic, male population. Am J Clin Nutr 1997;66:1323-31.

24. National Cancer Institute. Common terminology criteria for adverse events v3.0 (CTCAE). Bethesda, MD: NCI, 2003.

25. Ruppert D, Wand MP, Carroll RJ. Semiparametric regression. Cambridge, United Kingdom: Cambridge University Press, 2003:91-111, 356-7.

26. Schulz KF, Altman DG, Moher D, for the CONSORT Group. CONSORT 2010 statement: updated guidelines for reporting parallel group randomised trials. BMJ 2010;340:c332.

27. Neville KA, Cohn RJ, Steinbeck KS, Johnston K, Walker JL. Hyperinsulinemia, impaired glucose tolerance, and diabetes mellitus in survivors of childhood cancer: prevalence and risk factors. J Clin Endocrinol Metab 2006;1991:4401-7.

28. Chemaitilly W, Boulad F, Oeffinger KC, Sklar CA. Disorders of glucose homeostasis in young adults treated with total body irradiation during childhood: a pilot study. Bone Marrow Transplant 2009;44:339-43.

29. Streat SJ, Beddoe AH, Hill GL. Aggressive nutritional support does not prevent protein loss despite fat gain in septic intensive care patients. J Trauma 1987;27:262-6.

30. Geibig CB, Owens JP, Mirtallo JM, Bowers D, Nahikian-Nelms M, Tutschka P. Parenteral nutrition for bone marrow transplant recipients: evaluation of an increased nitrogen dose. JPEN J Parenter Enteral Nutr. 1991;15:184-8.

31. Bozzetti F, Gavazzi C, Mariani L, Crippa F. Artificial nutrition in cancer patients: which route, which composition? World J Surg 1999; 23:577-83.

32. Jarden M, Baadsgaard MT, Hovgaard DJ, Boesen E, Adamsen L. A randomized trial on the effect of a multimodal intervention on physical capacity, functional performance, and quality of life in adult patients undergoing allogeneic SCT. Bone Marrow Transplant 2009;43:725-37.

33. Chamorro-Viña C, Ruiz J, Santana-Sosa E, Gonzalez Vicent M, Madero L, Perez M, Ramirez M, Lucia A. Exercise during hematopoietic stem cell transplant hospitalization in children. Med Sci Sports Exerc 2010; 42:1045-53. 
34. Knols RH, de Bruin ED, Uebelhart D, Aufdemkampe G, Schanz U, Stenner-Liewen F, Hitz F, Taverna C, Aaronson NK. Effects of an outpatient physical exercise program on hematopoietic stem-cell transplantation recipients: a randomized clinical trial. Bone Marrow Transplant 2011;46:1245-55.

35. Choban PS, Burge JC, Scales D, Flancbaum L. Hypoenergetic nutrition support in hospitalized obese patients: a simplified method for clinical application. Am J Clin Nutr 1997;66:546-50.

36. Owais AE, Bumby RF, MacFie J. Permissive underfeeding in short-term nutritional support. Aliment Pharmacol Ther 2010;32:628-36.

37. Arabi YM, Tamim HM, Dhar GS, Al-Dawood A, Al-Sultan M, Sakkijha M, Kahoul SH, Britts R. Permissive underfeeding and intensive insulin therapy in critically ill patients: a randomized controlled trial. Am J Clin Nutr 2011;93:569-77.

38. Gooley TA, Chien JW, Pergam SA, Hingorani S, Sorror ML, Boeckh M, Martin PJ, Sandmaier BM, Marr KA, Appelbaum FR, et al. Reduced mortality after allogeneic hematopoietic-cell transplantation. N Engl J Med 2010;363:2091-101.
39. Chow EJ, Simmons JH, Roth CL, Baker KS, Hoffmeister PA, Sander JE, Friedman DL. Increased cardiometabolic traits in pediatric survivors of acute lymphoblastic leukemia treated with total body irradiation. Biol Blood Marrow Transplant 2010;16:1674-81.

40. Perkins JL, Kunin-Batson AS, Youngren NM, Ness KK, Ulrich KJ, Hansen MJ, Petryk A, Steinberger J, Anderson FS, Baker KS Long-term follow-up of children who underwent hematopoeitic cell transplant (HCT) for AML or ALL at less than 3 years of age. Pediatr Blood Cancer 2007;49:958-63.

41. Oeffinger KC, Mertens AC, Sklar CA, Kawashima T, Hudson MM Meadows AT, Friedman DL, Marina N, Hobbie W, Kadan-Lottick NS, et al. Chronic health conditions in adult survivors of childhood cancer. N Engl J Med 2006;355:1572-82.

42. Oeffinger KC, Mertens AC, Sklar CA, Yasui Y, Fears T, Stovall M, Vik TA, Inskip PD, Robison LL. Obesity in adult survivors of childhood acute lymphoblastic leukemia: a report from the Childhood Cancer Survivor Study. J Clin Oncol 2003;21:1359-65. 